\section{Stepped care in psychological therapies: access, effectiveness and efficiency}

\author{
Narrative literature review \\ PETER BOWER and SIMON GILBODY
}

\begin{abstract}
Background There is a significant gap between the demand for psychological therapy services and the available supply. One proposal to overcome this problem is to increase efficiency of provision through the adoption of briefer 'minimal interventions' within stepped care models.
\end{abstract}

Aims To examine the theoretical underpinnings of stepped care, together with the practicalities of the adoption of this system of care.

Method Narrative literature review.

Results The potential clinical and economic benefits of stepped care are dependent upon underlying assumptions of equivalence in terms of clinical outcomes, efficiency in terms of resource use and costs, and acceptability of 'minimal interventions' to patients and therapists. Illustrative studies of these issues are considered.

Conclusions Although psychological services might benefit from the adoption of the stepped care model, a substantial research agenda needs to be fulfilled before a judgement can be made as to whether stepped care might be an efficient method of delivering psychological services.

\section{Declaration of interest None.}

The clinical effectiveness of psychological therapy is generally accepted (Department of Health, 2001), but current focus is on issues of cost and the efficient use of limited psychological therapy resources in publicly funded or managed healthcare systems (Christensen \& Jacobson, 1994; Haaga, 2000; Lovell \& Richards, 2000; Newman, 2000; Scogin et al, 2003). At present psychological therapy services provide poor access, with only a minority of patients in need able to receive therapy (Lovell \& Richards, 2000) and the vast majority receiving no treatment (Bebbington et al, 2000). This is because the available treatment resource is limited by the relatively small number of trained therapists. A number of authors have recently suggested 'stepped care' as a solution (Kaltenthaler et al, 2002; Marks et al, 2003; National Collaborating Centre for Mental Health, 2003; Scogin et al, 2003). This review considers the meaning of stepped care in relation to psychological therapies, reviews the current evidence to support stepped care and outlines the future research agenda.

\section{STEPPED CARE MODELS}

Stepped care is a model of healthcare delivery with two fundamental features. First, the recommended treatment within a stepped care model should be the least restrictive of those currently available, but still likely to provide significant health gain. Second, the stepped care model is selfcorrecting.

The definition of 'least restrictive' may refer to the impact on patients in terms of cost and personal inconvenience (Sobell $\&$ Sobell, 2000), but in the context of publicly funded healthcare systems where specialist therapist time is a key limiting factor, 'least restrictive' is often interpreted as referring to the amount of specialist therapist time required (i.e. treatment intensity). In stepped care, more intensive treatments are generally reserved for people who do not benefit from simpler first-line treatments, or for those who can be accurately predicted not to benefit from such treatments (Newman, 2000). In this way, stepped care has the potential for deriving the greatest benefit from available therapeutic resources. However, the focus on low-intensity treatment delivery might actually be counterproductive, without the crucial self-correcting mechanism. 'Selfcorrecting' means that the results of treatments and decisions about treatment provision are monitored systematically, and changes are made ('stepping up') if current treatments are not achieving significant health gain. Rather than regarding withdrawal from therapy as a reflection of a lack of motivation for treatment on the part of the patient, stepped care models see monitoring of progress and outcome as a fundamental responsibility of therapists and the system of care within which they work. A simple two-step model is outlined in Fig. 1.

Although these features of stepped care may seem to resemble the way in which many clinicians implicitly operate (Breslin et al, 1997; King et al, 2002), stepped care standardises systems and procedures with an explicit aim of improving efficiency (Katon et al, 1997; Scogin et al, 2003). Stepped care models have been described in relation to smoking (Abrams et al, 1996; Smith et al, 2001), back pain (Von Korff \& Moore, 2001), alcohol treatment (Breslin et al, 1997; Sobell \& Sobell, 2000), migraine (Sculpher et al, 2002), anxiety (Newman, 2000), eating disorders (Wilson et al, 2000), methadone maintenance (King et al, 2002) and depression (Simon et al, 2001; Scogin et al, 2003).

\section{Key elements in stepped care models}

What treatments are of relevance to stepped care?

Stepped care requires treatments of differing intensity. This requirement is amply satisfied in psychological therapies, where a number of less intensive treatments (or 'minimal interventions') have been developed, for example brief therapies (Scott et al, 1997), group treatments (Dowrick et al, 2000) and self-help approaches such as bibliotherapy (Cuijpers, 1997) and computerised treatments (Proudfoot et al, 2004).

The main candidate for stepped care is cognitive-behavioural therapy. This therapy 


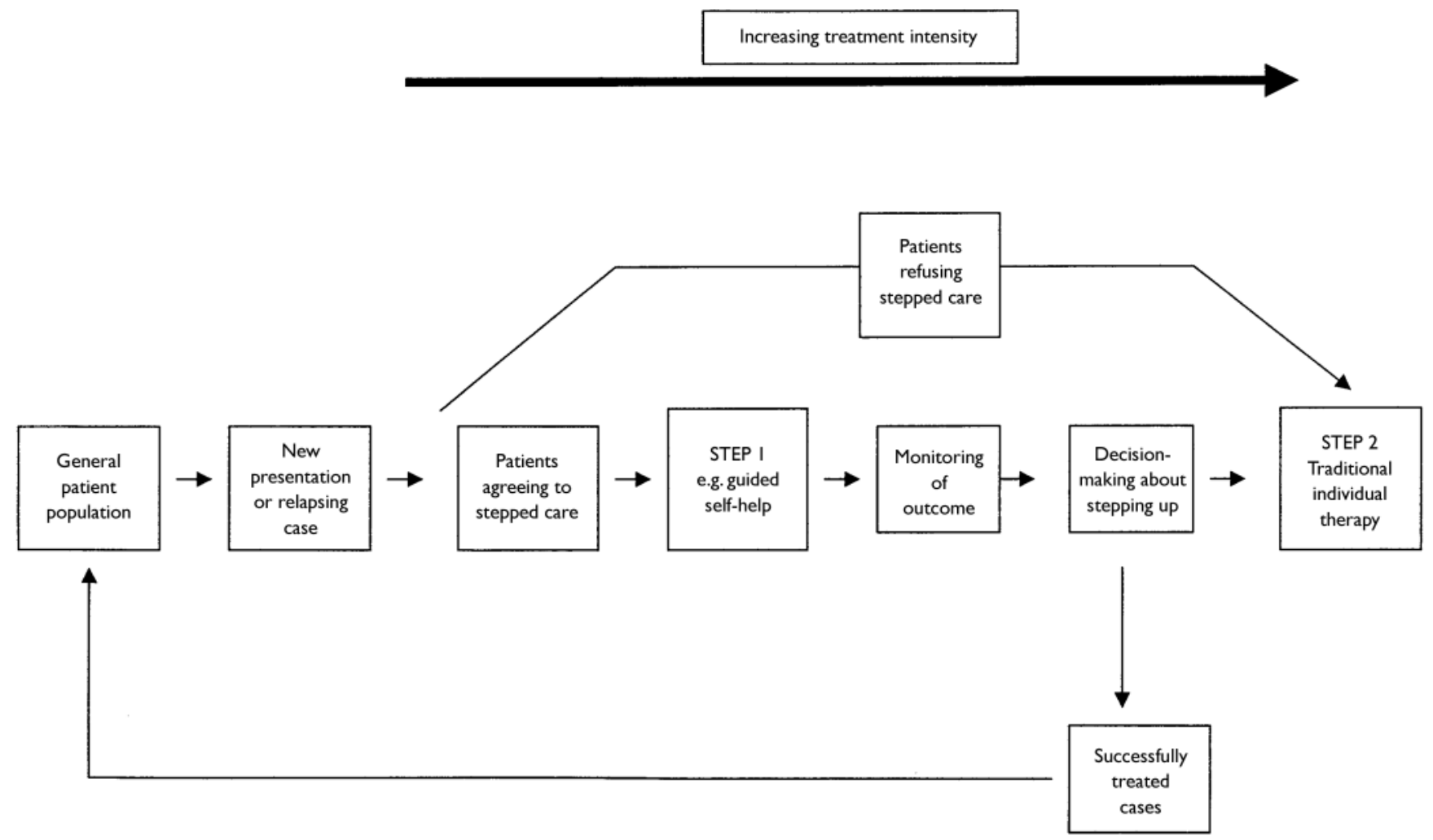

Fig. I Key elements in a simple stepped care model.

has been highlighted by guidelines as the treatment of choice for a number of disorders (Department of Health, 2001). It also has the advantage that there can be a degree of commonality between different 'steps', all being based on the same theoretical model and therapeutic techniques. There might be advantages to such consistency, although 'stepping up' to an approach that has already failed might be seen as counterproductive (Newman, 2000). However, there is no requirement for stepped care to involve a single therapeutic approach (Davison, 2000; Sobell \& Sobell, 2000). Other brief treatments such as problem-solving (Dowrick et al, 2000) are of proven benefit, and a stepped care model might involve 'stepping up' from cognitive-behavioural bibliotherapy to counselling (Ward et al, 2000) or interpersonal therapy (Schulberg et al, 1996). Different treatments may have an effect on different aspects of the patient's problem (Newman, 2000).

The issue of medication should also not be ignored. Generally, access to medication is not constrained in the same way as access to psychological therapy, because it does not generally require access to a specialist therapist. However, modern models of collaborative care do involve significant support from case managers to improve compliance with medication (Badamgarav et al, 2003), and thus the principles of stepped care can be applied in this context (Katon et al, 1999; Araya et al, 2003; Unutzer et al, 2003).

\section{How many steps should be involved?}

The number of steps will be bounded by two factors. First, there are only a finite number of interventions available that differ qualitatively in terms of intensity. Second, since stepped care seeks to reduce the average amount of therapeutic input per patient, an upper limit is provided by the amount of therapist input that is routinely provided in traditional services. In the UK National Health Service, this might be 8-16 sessions ( $50 \mathrm{~min}$ long) of face-to-face therapy, often cognitive-behavioural (Lovell \& Richards, 2000; Department of Health, 2001). Given this ceiling, there are probably four qualitatively different steps: pure self-help (no therapist input beyond assessment); guided self-help and group therapy (therapist input around 1-2 $\mathrm{h}$ per patient); brief individual therapy (therapist input around $6 \mathrm{~h}$ per patient); and longer-term individual therapy (therapist input around $16 \mathrm{~h}$ per patient). Different steps might use different professionals, with pure self-help at the initial step, followed by guided self-help from a practice nurse (Richards et al, 2002) or primary care mental health worker (Bower, 2002), then individual therapy from a clinical psychologist or mental health nurse.

Much informal mental healthcare is undertaken by patients in the community without the intervention of mental health services (Rogers et al, 1999), and the first level of any stepped care system might involve facilitating 'natural recovery' outside statutory services (Sobell \& Sobell, 2000). However, the relationship between such community-level initiatives and statutory services is likely to be complex (Abrams et al, 1996).

\section{How are decisions to be made about 'stepping up'?}

'Stepping up' requires a decision about patient progress based on judgements about 'significant health gain' or 'improvement', but the exact definition will be dependent on the type of disorder, its natural history and the effectiveness of available treatments 
(Breslin et al, 1997, 1999; Sobell \& Sobell, 2000). This might involve the use of 'clinically significant and reliable change' (Jacobson \& Truax, 1991) or individualised 'expected outcomes' based on patient pre-treatment variables (Lutz, 2003). In some disorders, different criteria might be relevant. For example, in depression, because residual symptoms are associated with later relapse, treatment until full remission has been recommended (Scogin et al, 2003).

Decisions may be based on data collected within treatment (Breslin et al, 1997). The use of simple assessment and decision rules (e.g. a threshold score on the Beck Depression Inventory completed by a patient) has the advantage that this approach is cheap and easily standardised (Scogin et al, 2003). More complex assessments by experienced clinicians might have advantages but would add significantly to costs, and it is important that the benefits of stepped care are not swallowed up by the professional time required to implement the system. Furthermore, professional decision-making may on occasion be less valid than judgements made on the basis of guidelines and decision rules (Breslin et al, 1997). There is also a trade-off between false positives (i.e. inappropriate 'stepping up') and false negatives (failure to 'step up' when appropriate), and the complexity of decision-making should therefore be sensitive to the costs of treatment at different steps (Sobell \& Sobell, 2000).

The role of the patient requires consideration, as choice of treatment might be an important component of patient-centred services, but might also have important implications for efficiency within stepped care. The whole issue of the acceptability of stepped care to patients is dealt with in more detail below.

\section{Which patients or disorders are appropriate for stepped care models?}

Stepped care models may be most appropriate for disorders in which adverse consequences would not result from starting patients on too low a step, or where the perception of 'treatment failure' at lower levels does not greatly affect later outcome. For example, in the treatment of eating disorders, stepped care might be far more appropriate for bulimia and binge eating than for anorexia nervosa (Wilson et al, 2000). A recent model for depression (Scogin et al, 2003) suggests that stepped care psychological therapy might be particularly relevant for minor depression, as opposed to major depression: the latter might be better managed through complex collaborative models of care (Gilbody et $a l, 2003)$. There is a general perception that minimal interventions are best restricted to less severe disorders, although the evidence for this is not definitive (Lovell \& Richards, 2000). However, it is important that decisions about the use of minimal interventions as a first-line treatment are made on the basis of good research evidence, as there may be cases in which early intensive treatment is actually more clinically effective and cost-effective than a minimal intervention.

\section{CLINICAL AND ECONOMIC EVALUATION OF STEPPED CARE MODELS}

Although the practical issues outlined above are important, current interest in stepped care is based on three fundamental assumptions:

(a) minimal interventions can provide 'significant health gain' equivalent to that of traditional psychological therapies, at least for a proportion of patients (equivalence assumption);

(b) using minimal interventions will therefore allow current healthcare resources to be used more efficiently (efficiency assumption);

(c) minimal interventions and the stepped care approach are acceptable to patients and professionals (acceptability assumption).

\section{Equivalence assumption}

There is evidence that minimal interventions can, like their more therapist-intensive versions, be more effective than 'usual care' or 'no treatment' conditions (Cuijpers, 1997; Dowrick et al, 2000; Lovell \& Richards, 2000; Bower et al, 2001; Kaltenthaler et al, 2002). However, such comparisons are not the most relevant to the equivalence assumption. First, they do not compare minimal interventions with traditional, more intensive versions. Furthermore, they may also validate the efficiency assumption. For example, if a simple bibliotherapy treatment is superior to 'no treatment', providing bibliotherapy to patients would still need an overall increase in the amount of resources required - or cutbacks in other parts of the service - even if the bibliotherapy were relatively cheap. Whether that is worthwhile is a question of allocative efficiency (Donaldson et al, 2002). Only when a treatment costs less and provides equal effectiveness is it described as technically efficient, which is the key criterion for stepped care treatments. Therefore, published demonstrations of the benefits of 'stepped care' treatments $v$. usual care may not be definitive in the current context.

The key demonstration required to support stepped care models is the comparison of minimal interventions with traditional psychological therapy. A number of studies have reported that minimal interventions do not differ in effectiveness from more intensive, therapist-delivered treatments (Lovell \& Richards, 2000). However, the finding that there is no difference in the clinical effectiveness of two treatments does not prove that they are equivalent. Most psychological therapy trials are designed and powered to examine whether there are differences in effectiveness, whereas proving equivalence requires specific analytical procedures (Rogers et al, 1996; Ebbutt $\&$ Frith, 1998) and routinely requires a larger sample size (Hargreaves et al, 1999; Durand \& King, 2003). A trial with a small sample size might conclude that two treatments did not differ significantly in a statistical sense, even though the outcomes differed in a clinically significant way.

This problem can be illustrated with respect to a recent systematic review of computerised cognitive-behavioural therapy. Six trials compared it with the therapist-led form of this therapy, and the review reported that five of them showed computerised therapy to be as good as the therapist-led treatment (Kaltenthaler et al, 2002). However, the sample sizes per group in these studies ranged from 6 to 38, which means that the finding of 'no statistically significant difference' between the two forms of delivery of cognitive-behavioural therapy is likely to occur even in the presence of important treatment effects. This can be illustrated most easily by summarising the data in terms of standardised effect sizes, rather than statistical significance (available from the author upon request). In four trials reporting 'no statistically significant differences' between treatments, 32 comparisons were made between computerised and therapist-led cognitivebehavioural therapy, and $9(28 \%)$ of these differences were either 'medium' or 'large' 
according to current conventions (Lipsey, 1990). This highlights the fact that small trials may report no significant difference between treatments that do in fact differ in clinically significant ways.

However, it should be noted that traditional equivalence trials examine equivalence at the level of the individual patient, whereas it is also possible to view this issue from the perspective of a population. For example, a modestly effective treatment that could be used with a large number of patients might provide more population health benefit than a more effective treatment that could only be provided to a small proportion of the population. An alternative trial design, the so-called outcome maximisation design (Sutton, 1997), seeks to determine how much overall clinical benefit can be derived from a given therapeutic resource. Such a design may be appropriate for testing whether traditional, intensive psychological therapies, which might be more clinically effective with individual patients, are also delivering greater benefits at the level of the population.

\section{Efficiency assumption}

Equivalent clinical outcomes in traditional and minimal interventions have been taken to imply that the latter are more efficient because of the lower amount of therapist input required. For example, studies in alcohol treatment have quoted data relating to 'therapist hours per abstinent patient' (Wilson et al, 2000). This may be valid from the perspective of a psychological therapy services manager. However, other stakeholders may require a more comprehensive analysis of costs, such as direct treatment costs in other sectors (e.g. primary care visits and medication, use of social services and voluntary sector providers) and patient costs (such as purchasing manuals or using private therapy). Limiting the analysis of costs to those associated with the psychological therapy service alone ignores the potential for cost-shifting to other sectors. Demonstrations of increased efficiency in stepped care models may be illusory if significant cost-shifting occurs. There is some evidence that patients receiving minimal interventions are more likely to seek additional interventions than those receiving traditional treatments (Treasure et al, 1996; Thiels et al, 1998).

\section{Acceptability assumption}

Patient attitudes to psychological therapy are generally very positive (Priest et al, 1996), but it cannot be assumed that these positive attitudes will generalise to minimal interventions. Patients may feel that the provision of minimal interventions is inappropriate (Scogin et al, 2003), especially for more severe disorders (Landreville et al, 2001). Trials of minimal interventions in primary care rarely report overall uptake rates to the offer of such treatment (Bower et al, 2001). One non-randomised study found that one-fifth of patients motivated to return a screening questionnaire to a computerised self-help clinic refused computer-aided treatment when it was offered (Marks et al, 2003). In another study, scarcely more than half of patients offered self-help while on a waiting list for cognitive-behavioural therapy took up the offer (Whitfield et al, 2001).

The issue of patient acceptability raises the possibility of tension between the desire to gain the greatest amount of clinical benefit from available resources, and the need to provide services that are patient-centred and meet current policy concerns with providing choice to patients. Clearly, the goal is to allow patients to have a role in treatment decision-making, and there are published trials in which psychological therapy and pharmacotherapy treatments were both available within a stepped care model, and patients could choose between them in collaboration with their clinicians (Unutzer et al, 2003). Equally, within a stepped care system such as that outlined in Fig. 1, patients may be provided with a choice of interventions within steps (for example, guided self-help, computerised cognitive-behavioural therapy or group therapy at step 1), even if choices between steps are more constrained. However, the efficiency benefits of stepped care may be determined in part by constraints on choice: for example, offering minimal interventions such as bibliotherapy at the first step may have little effect if patients are allowed to choose more intensive treatment, and if most have an expectation of (and preference for) the latter type of treatment.

A second important issue is the acceptability of stepped care to professionals. Trials may be conducted by advocates of minimal intervention, and it cannot be assumed that all professionals will feel comfortable with the philosophical assumptions that underpin stepped care models or the changes to working methods that may be required (Cushman \& Gilford, 2000).

\section{Equivalence, efficiency and acceptability assumptions in published 'stepped care' trials}

There are few studies of a complete stepped care model. Two relevant studies of stepped care for eating disorders have been conducted in the UK (Treasure et al, 1996; Palmer et al, 2002), and one is considered here in detail to highlight important issues relating to the equivalence, efficiency and acceptability assumptions. This trial compared standard 16-session cognitivebehavioural therapy for bulimia nervosa with a stepped care model, in which the first step was the use of a self-help manual for 8 weeks (Treasure et al, 1996). After this period patients were assessed, and those who no longer met ICD-10 criteria for bulimia were not offered further therapy, whereas those who did were offered eight sessions of cognitive-behavioural therapy. Sample size at baseline was 55 per group. In the stepped care arm, 16 out of 41 patients $(39 \%)$ were deemed to be recovered at 8 weeks and were not offered further therapy. Of the remaining patients, 9 were offered treatment but did not take it up. There was an $11 \%$ difference in withdrawal rates in favour of the stepped care group. In terms of outcomes, end-of-treatment abstinence rates in both groups were $30 \%$, and at 18 months remission was $40 \%$ in the stepped care group and $41 \%$ in the traditional treatment group. Outside the trial, $38 \%$ of patients in the stepped care group sought extra treatment, compared with $17 \%$ of the traditional treatment group.

The trial does highlight some of the problems in providing a rigorous evidence base for stepped care. First, it should be noted that none of the main results listed (relating to withdrawal from the trial, other treatment or clinical outcome) were reported as being statistically significant, although the differences reported in withdrawal rates and treatment outside the trial may be important clinically and economically. This highlights the problems of demonstrating equivalence when the sample size is relatively modest. The trial did provide some evidence of similar outcomes in the less intensive treatment arm, and implementation would allow more patients to be treated from the same 
therapeutic resources. However, this evidence of efficiency was restricted to the psychological therapy service, and there were no data on where the 'extra treatment' was sought and where the costs fell (e.g. on the wider healthcare system or the patient). Also, the costs of the 'stepping up' assessment were not taken into account. No specific data were presented on the acceptability of the model, although the rates of withdrawal suggest that the manual was generally acceptable to patients.

\section{FUTURE RESEARCH AGENDA}

The acceptability of stepped care is crucial for effective implementation; however, there is little information about this. Research on the patient acceptability assumption would need to access the views of a number of different populations, including not only those attending traditional psychological therapy services (who may be the first to enter stepped care models) but also those in primary care and the wider community, who currently do not access services but might be able to, if more efficient services were implemented. The views of these patients may differ in important ways (Meltzer et al, 2000).

Given the concerns discussed above about current evidence on the equivalence and efficiency assumptions, the initial research agenda needs to focus on rigorous evaluations of these assumptions in the comparison of a selection of current minimal interventions (such as bibliotherapy and computerised treatments) with traditional psychological therapy. Such studies would provide evidence concerning the components of the stepped care model, but not about the model as a whole. Observational evaluations of a functioning stepped care model could be used to examine issues such as patient throughput within the various steps, issues relating to decisionmaking about 'stepping up' and possible negative effects of treatment failure at lower steps and loss of continuity caused by changes in providers. As noted above, economic benefits might be dependent on patients restricting their help-seeking to the minimal interventions provided, and qualitative research to understand patient decision-making would be useful (Pilgrim et al, 1997; Breslin et al, 1999).

The final stage in the research agenda would be a controlled trial evaluation to compare an entire stepped care model with a traditional model of service delivery. This might require a large-scale cluster randomised trial (Gilbody \& Whitty, 2002), and would seek to quantify the overall public health benefit of traditional and stepped care models.

However, the time required to complete such an ambitious research agenda may significantly delay service development. A more efficient alternative might be the use of modelling, which refers to the use of mathematical simulations to assess outcomes and costs associated with different health technologies and patient populations (Chilcott et al, 2003). Modelling could identify the potential for stepped care to be both clinically effective and costeffective based on current data; in addition, models might be useful to extend results from existing randomised controlled trials and in the design of future trials (Chilcott et al, 2003).

First, modelling could be used to extend current trial data by examining longer-term outcomes (e.g. relapse and recurrence), different comparators, the effect of context (e.g. US data in the UK setting) and the effects of minimal interventions within a broader system of care (Brennan \& Akehurst, 2000). For example, modelling could be used to determine the optimal number of steps in a stepped care model (Fig. 1), by identifying the number that provided the best potential trade-off between efficiency and complexity. Costeffectiveness within a model could be examined from a wider societal perspective than is the case with existing research. Data on the effects of different interventions in the hierarchy of stepped care could be combined to examine pathways through the model of a hypothetical cohort of people with depression (Valenstein et al, 2001). The effect of the patient acceptability assumption could be modelled by examining the effect of assuming that different proportions of patients refuse to enter the lower levels of the hierarchy and enter more intensive steps.

Second, modelling could assist in informing the design of future trials and indicating their likely value. Models could help to specify an appropriate equivalence limit for future comparisons of minimal interventions and traditional therapy, suggest the optimal length of follow-up of outcomes (Chilcott et al, 2003) and highlight the key parameters that determine the likely cost-effectiveness of stepped care, and thus assist in the prioritisation of future trials (Torgerson \& Byford, 2002).

\section{CONCLUDING REMARKS}

Stepped care models have potential to improve the efficiency of psychological therapy provision. However, the optimal content and organisation of stepped care is as yet unclear. Although there is some supportive evidence for the use of stepped care, rigorous evaluations of the underlying assumptions are scarce, and a significant research agenda remains. Modelling may be a useful research method in the shorter term.

\section{REFERENCES}

Abrams, D., Orleans, C., Niaura, R., et al (1996) Integrating individual and public health perspectives for treatment of tobacco dependence under managed health care: a combined stepped care and matching model. Annals of Behavioral Medicine, I8, 290-304.

Araya, R., Rojas, C., Fritsch, R., et al (2003) Treating depression in primary care in low income women in Santiago, Chile: a randomised controlled trial. Lancet, 361, 995-1000.

Badamgarav, E., Weingarten, S., Henning, J., et al (2003) Effectiveness of disease management programs in depression: a systematic review. American Journal of Psychiatry, 160, 2080-2090.

Bebbington, P., Brugha, T., Meltzer, H., et al (2000)

Neurotic disorders and the receipt of psychiatric treatment. Psychological Medicine, 30, 1369-1376.

Bower, P. (2002) Primary care mental health workers: models of working and evidence of effectiveness. British Journal of General Practice, 52, 926-933.

Bower, P., Richards, D. \& Lovell, K. (200I) The clinical and cost-effectiveness of self-help treatments for anxiety and depressive disorders in primary care: a systematic review. British Journal of General Practice, 5I 838-845.

Brennan, A. \& Akehurst, R. (2000) Modelling in health economic evaluation: what is its place? What is its value? Pharmacoeconomics, 17, 445-459.

Breslin, F., Sobell, M., Sobell, L., et al (1997) Towards a stepped care approach to treating problem drinkers: the predictive utility of within-treatment variables and therapist prognostic ratings. Addiction, 92, 1479-1489.

Breslin, F., Sobell, M., Sobell, L., et al (1999) Problem drinkers: evaluation of a stepped-care approach. Journal of Substance Abuse, 10, 217-232.

Chilcott, J., Brennan, A., Booth, J., et al (2003) The role of modelling in the prioritising and planning of clinical trials. Health Technology Assessment, 7 (23).

Christensen, A. \& Jacobson, N. (1994) Who (or what) can do psychotherapy? The status and challenge of nonprofessional therapies. Psychological Science, 5, 8-14.

Cuijpers, P. (1997) Bibliotherapy in unipolar depression: a meta-analysis. Journal of Behaviour Therapy and Experimental Psychiatry, 28, 139-147.

Cushman, P. \& Gilford, P. (2000) Will managed care change our way of being? American Psychologist, 55, 985-996. 
Davison, G. (2000) Stepped care: doing more with less? Journal of Consulting and Clinical Psychology, 68, 580-585.

Department of Health (200I) Treatment Choice in Psychological Therapies and Counselling: Evidence Based Clinical Practice Guideline. London: Department of Health.

Donaldson, C., Currie, G. \& Mitton, C. (2002) Cost effectiveness analysis in health care: contraindications. BMJ, 325, 89I-894.

Dowrick, C., Dunn, G., Ayuso-Mateos, J.-L., et al (2000) Problem solving treatment and group psychoeducation for depression: multicentre randomised controlled trial. BMJ, 32I, I-6.

Durand, A. \& King, M. (2003) Specialist treatment versus self-help for bulimia nervosa: a randomised controlled trial in general practice. British Journal of General Practice, 53, 37I-377.

Ebbutt, A. \& Frith, L. (1998) Practical issues in equivalence trials. Statistics in Medicine, 47, |69|-I70I.

Gilbody, S. \& Whitty, P. (2002) Improving the delivery and organisation of mental health services: beyond the conventional randomised controlled trial. British Journal of Psychiatry, 180, 13-18.

Gilbody, S., Whitty, P., Grimshaw, J., et al (2003) Educational and organisational interventions to improve the management of depression in primary care: a systematic review. JAMA, 289, 3145-3I5I.

Haaga, D. (2000) Introduction to the special section on stepped care models in psychology. Journal of Consulting and Clinical Psychology, 68, 547-548.

\section{Hargreaves, W., Shumway, M. \& Hu, T. (1999)}

Measuring psychotherapy cost and effectiveness. In Costeffectiveness of Psychotherapy: A Guide for Practitioners, Researchers and Policy Makers (eds N. Miller \& K Magruder), pp. 85-98. New York: Oxford University Press.

Jacobson, N. \& Truax, P. (199I) Clinical significance: a statistical approach to defining meaningful change in psychotherapy research. Journal of Consulting and Clinica Psychology, 59, 12-19.

Kaltenthaler, E., Shackley, P., Stevens, P., et al (2002) A systematic review and economic evaluation of computerised cognitive behaviour therapy for depression and anxiety. Health Technology Assessment, 6(22).

Katon, W., Von Korff, M., Lin, E., et al (1997) Population-based care of depression: effective disease management strategies to decrease prevalence. General Hospital Psychiatry, 19, 169-178.

Katon, W., Von Korff, M., Lin, E., et al (1999) Stepped collaborative care for primary care patients with persistent symptoms of depression: a randomized trial. Archives of General Psychiatry, 56, II09-1II5.

King, V., Stoller, K., Hayes, M., et al (2002) A multicenter randomized evaluation of methadone medical maintenance. Drug and Alcohol Dependence, $\mathbf{6 5}$ 137-148.

Landreville, P., Landry, J., Baillargeon, L., et al (200I) Older adults' acceptance of psychological and pharmacological treatments for depression. Journal of Gerontology: Psychological Sciences, 56B, 285-29.

Lipsey, M. (1990) Design Sensitivity: Statistical Power for Experimental Research. Newbury Park: Sage.

Lovell, K. \& Richards, D. (2000) Multiple Access Point and Levels of Entry (MAPLE): ensuring choice, accessibility and equity for CBT services. Behavioral and Cognitive Psychotherapy, 28, 379-391.

\section{CLINICAL IMPLICATIONS}

- Traditional models of the delivery of psychological therapy cannot meet current demand. Other forms of delivery, such as brief therapies, group treatments and selfhelp, may provide useful alternatives.

Delivering alternative forms of psychological therapy through a stepped care model has significant potential to improve the efficiency of psychological therapy and ensure good outcomes for patients.

- There is only preliminary suppor tive evidence concerning stepped care systems in psychological therapies.

\section{LIMITATIONS}

- Crucial research questions remain to be addressed in relation to the clinical and economic assumptions underlying stepped care models.

- The acceptability of stepped care models to patients and professionals is unclear.

- The exact nature of the optimal stepped care model is also unclear, in terms of the number of steps, the treatments available at each step and the decision-making processes to determine when patients move between steps.

PETER BOWER, PhD, National Primary Care Research and Development Centre, University of Manchester; SIMON GILBODY, DPhil, MRCPsych, Academic Unit of Psychiatry, University of Leeds, UK

Correspondence: Dr Peter Bower, National Primary Care Research and Development Centre, 5 th Floor,Williamson Building, University of Manchester, Manchester MI3 9PL, UK. Tel: 0I6I 2757638 ; e-mail: peter.bower@man.ac.uk

(First received 13 November 2003, final revision 9 September 2004, accepted 10 September 2004)

Lutz, w. (2003) Efficacy, effectiveness, and expected treatment response in psychotherapy. Journal of Clinical Psychology, 59, 745-750.

Marks, I. M., Mataix-Cols, D., Kenwright, M., et al (2003) Pragmatic evaluation of computer-aided self-help for anxiety and depression. British Journal of Psychiatry 183, 57-65.

Meltzer, H., Bebbington, P., Brugha, T., et al (2000) Reluctance to seek treatment for neurotic disorders. Journal of Mental Health, 9, 319-327.

\section{National Collaborating Centre for Mental Health} (2003) Depression - Core Interventions in the Management of Depression in Primary and Secondary Care (National Clinical Practice Guideline - Draft for Consultation). London: National Institute for Clinical Excellence.

Newman, M. (2000) Recommendations for a cost offset model of psychotherapy allocation using generalized anxiety disorder as an example. Journal of Consulting and Clinical Psychology, 68, 549-555.

Palmer, R. L., Birchall, H., McGrain, L., et al (2002) Self-help for bulimic disorders: a randomised controlled trial comparing minimal guidance with face-to-face or telephone guidance. British Journal of Psychiatry, 18I, 230-235.

Pilgrim, D., Rogers, A., Clarke, S., et al (1997) Entering psychological treatment: decision making factors for GPs and service users. Journal of Interprofessional Care, II, 313-323.

Priest, R., Vize, C., Roberts, A., et al (1996) Lay people's attitudes to treatment of depression: results of opinion poll for Defeat Depression Campaign just before its launch. $B M \mid, \mathbf{3} \mathbf{3}, 858-859$.

Proudfoot, J., Ryden, C., Everitt, B., et al (2004) Clinical efficacy of computerised cognitive-behavioura therapy for anxiety and depression in primary care: randomised controlled trial. British Journal of Psychiatry, I85, 46-54.

Richards, D., Richards, A., Barkham, M., et al (2002) PHASE: a 'health technology' approach to psychological treatment in primary mental health care. Primary Health Care Research and Development, 3, 159-168.

Rogers, J., Howard, K. \& Vessey, J. (1996) Using significance tests to evaluate equivalence between two experimental groups. Psychological Bulletin, II3, 553-656.

Rogers, A., Hassell, K. \& Nicolaas, G. (1999) Demanding Patients? Analysing the Use of Primary Care. Milton Keynes: Open University Press.

Schulberg, H., Block, M., Madonia, M., et al (1996) Treating major depression in primary care practice: eight month clinical outcomes. Archives of General Psychiatry, 53, 913-919. 
Scogin, F., Hanson, A. \& Welsh, D. (2003) Selfadministered treatment in stepped-care models of depression treatment. Journal of Clinical Psychology, 59, 34I-349.

Scott, C., Tacchi, M. J., Jones, R., et al (1997) Acute and one-year outcome of a randomised controlled trial of brief cognitive therapy for major depressive disorder in primary care. British Journal of Psychiatry, I7I, 131-134.

Sculpher, M., Millson, D., Meddis, D., et al (2002)

Cost-effectiveness analysis of stratified versus stepped care strategies for acute treatment of migraine.

Pharmacoeconomics, 20, 91-100.

Simon, G., Katon, W., Von Korff, M., et al (200I) Costeffectiveness of a collaborative care program for primary care patients with persistent depression. American Journal of Psychiatry, 158, 1638-1644.

Smith, S., Jorenby, D., Fiore, M., et al (2001) Strike while the iron is hot. Can stepped care treatments resurrect relapsing smokers? Journal of Consulting and Clinical Psychology, 69, 429-439.
Sobell, M. \& Sobell, L. (2000) Stepped care as a heuristic approach to the treatment of alcohol problems. Journal of Consulting and Clinical Psychology, 68, 573-579.

Sutton, M. (1997) How to get the best health outcome for a given amount of money. BMJ, 315, 47-49.

Thiels, C., Schmidt, U., Treasure, J., et al (1998) Guided self change for bulimia nervosa incorporating use of a self-care manual. American Journal of Psychiatry, I55, 947-953.

Togerson, D. \& Byford, S. (2002) Economics notes: economic modelling before clinical trials. BMJ, 325, 98

Treasure, J., Schmidt, U., Troop, N., et al (1996)

Sequential treatment for bulimia nervosa incorporating a self-care manual. British Journal of Psychiatry, 168, 94-98.

Unutzer, J., Katon, W., Callahan, C., et al (2003)

Collaborative care management of late-life depression in the primary care setting: a randomized controlled trial. JAMA, 288, 2836-2845.
Valenstein, M., Vijan, S., Zeber, J., et al (200I) The cost-utility of screening for depression in primary care. Annals of Internal Medicine, 134, 345-360.

Von Korff, M. \& Moore, J. (200I) Stepped care for back pain: activating approaches for primary care. Annals of Internal Medicine, 134, 911-917.

Ward, E., King, M., Lloyd, M., et al (2000)

Randomised controlled trial of non-directive counselling, cognitive-behaviour therapy and usual GP care for patients with depression. I: Clinical effectiveness. BMJ, 32I, I383-1388.

Whitfield, G., Williams, C. \& Shapiro, D. (200I) Assessing the take up and acceptability of a self-help room used by patients awaiting their initial outpatient appointment. Behavioral and Cognitive Psychotherapy, 29, 333-343.

Wilson, G.,Vitousek, K. \& Loeb, K. (2000) Stepped care treatment for eating disorders. Journal of Consulting and Clinical Psychology, 68, 564-572. 